\title{
Raman spectroscopy reveals lipids in protein-containing SMA-stabilized lipodiscs
}

Maria Karlova $^{1}$, Dmitry Bagrov ${ }^{2}$, Maria Vorobyova ${ }^{3}$, Kahramon Mamatkulov $^{3}$, Grigory Arzumanyan ${ }^{3}$, Olga Sokolova ${ }^{1}$ and Konstantin Shaitan ${ }^{4}$

${ }^{1}$ Moscow Lomonosov University, United States, ${ }^{2}$ Lomonosov Moscow University, Moscow, Moskva, Russia, ${ }^{3}$ Department of Raman spectroscopy, Lab. Neutron Physics, Joint Institute for Nuclear Research, United States, ${ }^{4}$ Moscow Lomonosov State University, United States

Voltage-gated cation Kv7.1 channels, encoded with the KCNQ1 genes are a vital element in the formation of the cardiac action potential, and any functional alterations are accompanied by serious diseases. The activity of Kv7.1, like many other membrane proteins, is modulated by its surrounding lipids [1]. The removal of these lipids during purification can lead to structural changes and functional disorders. This is a significant drawback of the traditional approach to the extraction and purification of membrane proteins using detergents, their combinations, or mixtures with lipids. One of the modern methods for isolating membrane proteins is the use of a styrene-maleic acid (SMA) copolymer. This amphipathic copolymer can integrate into biological membranes and easily destroy them. As a result, discoid membrane fragments with a size of 10-40 nm are formed, surrounded by a copolymer belt [2]. Such particles are known as SMALPs (SMA lipid particles) or lipodiscs. The polymer has no affinity to any specific lipids, and, in SMALPs, the ratio of lipids remains the same as it was in the original membrane [3]. If the membrane contains proteins, they are enclosed into the forming lipodiscs, upon the addition of the copolymer. The ability to extract proteins with SMA has been demonstrated for liposomes [4], cell membrane fractions [5], and whole cells [6]. The SMA-extracted membrane proteins are quite stable and can be purified and furtheranalyzed by various biochemical methods $[5,6]$. The benefit of SMA-extraction is the possibility of completely avoiding detergents in the protein purification process. It means better preservation of the native conformation and lipid microenvironment of the proteins. The preservation of the lipid microenvironment was demonstrated for a number of SMA-extracted proteins using thin-layer chromatography and mass-spectrometry [7, 8]. However, these methods are indirect and require thepreliminary extraction of lipids from samples using mixtures of organic solvents with various additives. Presently, there is no universal extraction method that would be equally well suited for all lipid classes. Thus, it is necessary to select aspecific methodology for the target class. Often, the additives in the extraction mixtures are mutually exclusive, like acids for anionic lipid extraction and alkalis for sphingolipid extraction. Also, in some cases, additives can lead to degradation of the sample and to the appearance of artifacts. Here, we used mass-spectroscopy (LC-MS) and Raman spectroscopy to analyze the presence of lipids in the lipodisc stabilizing the recombinant human Kv7.1 protein. MS confirmed that the lipids were present in the lipodiscs, and Raman spectroscopy detected several spectral peaks, which could be attributed to lipids. The recombinant human Kv7.1 protein was expressed in the HEK cell line. Cells were lysed directly by the SMA solution. The lysate was clarified by centrifugation, and the supernatant was applied to the anti-V5 affinity column. TheKv7.1 protein was eluted with the V5 peptide. Protein expression and purification stages were controlled by SDS-PAGE and immunoblotting. Elution fractions containing the Kv7.1 protein were combined and subjected to lipid extraction using a modified Folch method [7]. Lipid composition was analyzed using LC-MS with electrospray ionization. According to MS, the sample contained triacylglycerols and diacylglycerols, phosphocholines, phosphoethanolamines, ceramides, and sphingomyelin. Unlike MS, Raman spectroscopy can be applied to intact lipodiscs. The system contains three chemically different components (SMA, Kv7.1, and lipids), and many of their Raman peaks overlap. So, it is difficult to attribute the observed peaks (Figure 1) to the vibrations of the chemical bonds of a specific component of the system. The Raman shift at $\sim 1000 \mathrm{~cm}-1$ can be attributed to either phenylalanine in Kv7.1, styrene in SMA, or C-H stretching in any of the three components. However, the peak in the fingerprint region centered at $1452 \mathrm{~cm}-1(-\mathrm{CH} 2$ 
scissoring mode) and inherently strong peaks in the region of $\sim 2800-3000 \mathrm{~cm}-1$ indicate the presence of lipids and confirm the data obtained using MS. Thus, we have shown that when the recombinant Kv7.1 protein is isolated using SMA, it retains lipids around the protein molecules. The Raman spectroscopy study of lipodiscs is still in progress. This work was supported by the Russian Foundation for Basic Research (RFBR) (Projects No. 18-504-12045 and No. 20-54-15004). Mass-spectrometry analysis was performed at the Advanced Mass Spectrometry Core Facility (AMS) in the Skolkovo Institute of Science and Technology, Moscow, Russia. D.V.B., O.S.S., K.V.S. acknowledge the support from the Interdisciplinary Scientific and Educational School of Moscow Lomonosov University «Molecular Technologies of the Living Systems and Synthetic Biology»

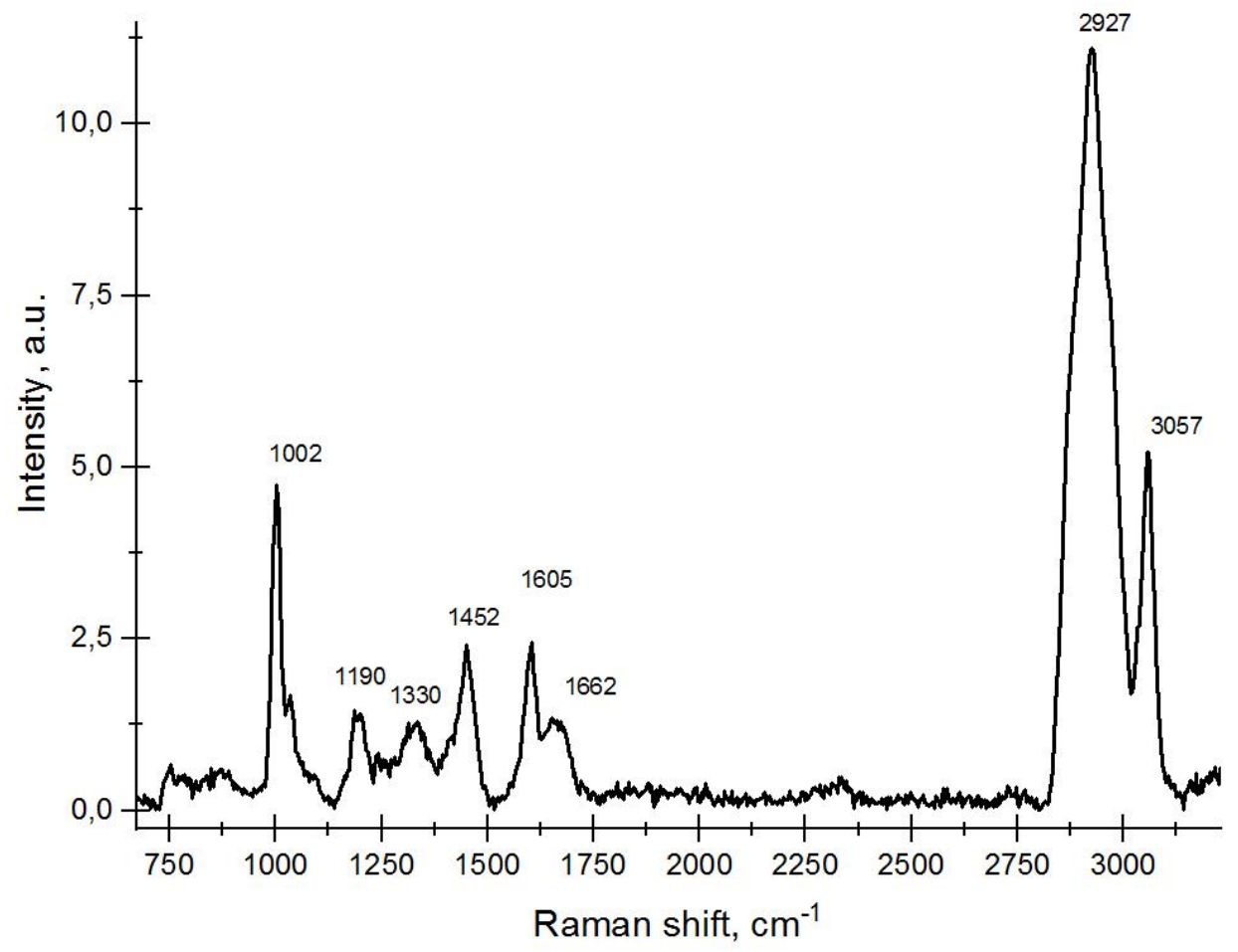

Figure 1. Raman spectra from Kv7.1 containing SMALPs, measured with $473 \mathrm{~nm}$ excitation.

\section{References}

1. Loussouarn G, Park KH, Bellocq C, Baró I, Charpentier F, Escande D. EMBO J. 2003;22(20):5412-5421. doi:10.1093/emboj/cdg526

2. Tonge SR, Tighe BJ. Adv Drug Deliv Rev. 2001;53(1):109-122. doi:10.1016/s0169-409x(01)00223-x

3. Dominguez Pardo JJ, Dörr JM, Iyer A, et al. Eur Biophys J. 2017;46(1):91-101. doi:10.1007/s00249-0161181-7

4. Knowles TJ, Finka R, Smith C, Lin YP, Dafforn T, Overduin M. J Am Chem Soc. 2009;131(22):7484-7485. doi:10.1021/ja810046q

5. Gulati S, Jamshad M, Knowles TJ, et al. Biochem J. 2014;461(2):269-278. doi:10.1042/BJ20131477

6. Karlova MG, Voskoboynikova N, Gluhov GS, et al. Chem Phys Lipids. 2019; 219:50-57. doi:10.1016/j.chemphyslip.2019.01.013

7. Teo ACK, Lee SC, Pollock NL, et al. Sci Rep. 2019;9(1):1813. Published 2019 Feb 12. doi:10.1038/s41598018-37962-0

8. Schmidt V, Sidore M, Bechara C, Duneau JP, Sturgis JN. BiochimBiophys Acta Biomembr. 2019;1861(2):431-440. doi:10.1016/j.bbamem.2018.10.017 